\title{
VLF Utilization at NASA Satellite Tracking Stations
}

\author{
C. H. Looney, Jr. \\ Contribution From Goddard Space Flight Center, Greenbelt, Md.
}

\begin{abstract}
The time and frequency requirements of the network of NASA satellite tracking stations are described, and it is shown that the VLF transmissions from WWVL satisfy many of these. There are great potentialities inherent in the use of these signals, or suitable modification of them, for obtaining extremely accurate standard time synchronizations. NASA tracking stations will greatly extend their usage of VLF reception in the near future, not only to obtain automatic frequency corrections, but for purposes of research in the area of time and frequency dissemination.
\end{abstract}

This paper describes, briefly, the network of stations, and their purpose, which serves as the reason for NASA's interest in the field of VLF frequency (and time) transmissions. Figure 1 shows the location of the Minitrack Stations which constitute the tracking network for unmanned satellites. These stations are required to maintain interstation time synchronization to $1 \mathrm{msec}$ in order to meet orbital accuracies imposed as computer requirements by the satellite experimenters. The stations used to track the manned orbiters do not as yet have accuracy requirements as stringent as those for the unmanned satellites. Stations for the purpose of acquiring telemetry data have the least stringent requirement for timing accuracy of any of the NASA Stations. These data acquisition stations are generally equipped with the same type time standards as are used in the tracking stations; however, the timing accuracy requirement is seldom less than 0.01 sec. NASA is presently installing three range and range rate tracking stations which will significantly improve tracking data and increase timing accuracy requirements. Later this year, a satellite with the code designation S-66 will be launched and will provide optical corner reflectors to be used in developing laser tracking methods. It appears that these optical methods will impose timing requirements of less than $100 \mu$ sec for interstation synchronization because of their potential tracking accuracies.

NASA has been interested in the properties of VLF which make it useful as a frequency standard device for several years and have monitored WWVL since it began transmissions in April 1960. Their first efforts were expended primarily in proving that reliable reception of the very few watts of WWVL's radiated power was possible. It has been particularly gratifying to discover that, with proper care in instrumentation, not only can the signal be received, but worldwide frequency measurements can be made to accuracies approaching a part in $10^{11}$. In the process of developing techniques capable of these accuracies, certain factors appear important. First, the transmitted frequency must be as accurate and invariant as possible. Excursions of even a few parts in $10^{11}$ should be avoided even though the average frequency might be the desired value. Second, the receiver must be so designed as to maintain the signal-to-noise ratio available at the antenna. Some receivers, which are quite good in high signalto-noise ratio environments, are virtually worthless for use with signal levels such as WWVL's $13 \mathrm{w}$ provided in the Washington, D.C., area. These receivers generally use frequency doubling techniques rather than the more conventional TRF or superheterodyne designs and lose signal-to-noise ratio by enhancing noise in the process of fraquency doubling. Both TRF and superheterodyne receivers in the laboratories in Greenbelt, Md., have been successfully used when the frequency doubling receivers indicated that no signals were present. And finally, careful techniques in reducing the records to numerical data must be employed. Use of more than one VLF station, independent checking of data reduction, and observation of pattern repetition from day to day have been the key factors in maintenance of con sistently accurate measurements.

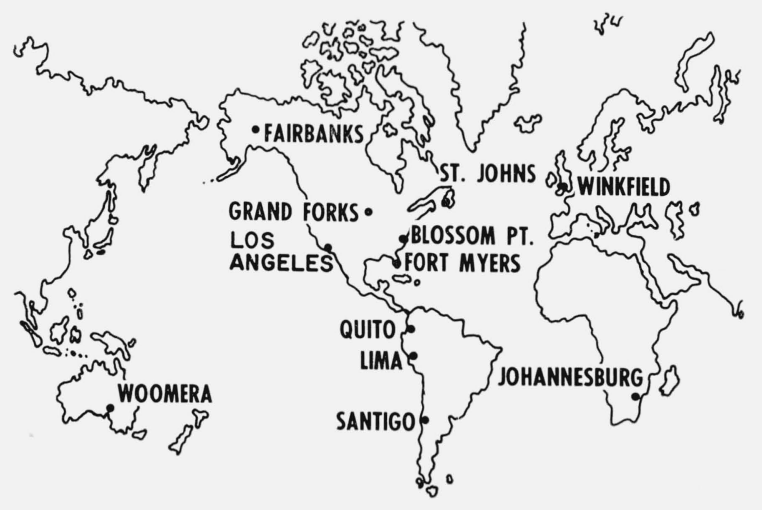

Figure 1. Map of present network stations. 


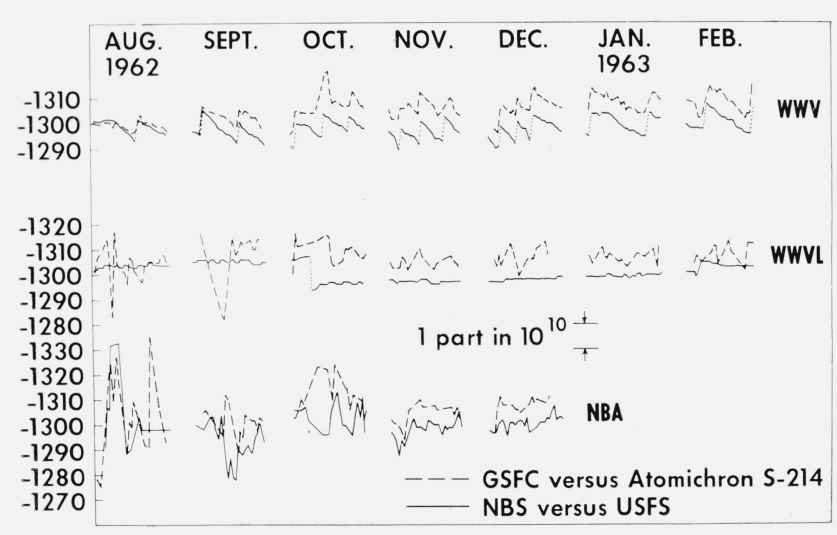

Figure 2. Frequency measurement.

Figure 2 is a comparison of data taken at NBS here in Boulder, with data taken at Goddard Space Flight Center in Greenbelt, Md. The GSFC measurements of WWV are made by direct comparison with a cesium beam oscillator's $5 \mathrm{mc} / \mathrm{s}$ output and are 1-hr measurements taken daily from 1200 to 1300 hours EST. The WWV antennas are less than 1,000 ft from the laboratory, so there are excellent conditions for noise-free measurements. NBS, on the other hand, must depend upon $20 \mathrm{kc} / \mathrm{s}$ and $60 \mathrm{kc} / \mathrm{s}$ measurements made at the WWV transmitter near Greenbelt, Md., and use smoothing techniques to reduce the effects of noise in relating the frequency of WWV to that of the United States Frequency Standard (USFS). The situation is reversed for WWVL, since NBS has precise knowledge of the frequency and phase angle of WWVL due to its proximity to the Boulder Laboratories; however, long integration times must be used at GSFC in order to receive the signal, and $24-\mathrm{hr}$ measurements are used to determine its frequency.

It is worth noting that the automatic phase correction of WWVL as received in Boulder, makes WWVL the most accurate of all the VLF frequency standard stations by removing phase changes due to antenna tuning variations and by removing the inherent limitations imposed by the use of a reference crystal oscillator.

Perhaps the most independent comparison of measurement techniques between NBS and GSFC is that obtained with radio station NBA. Both sites are equally situated here with respect to signal-to-noise ratio and the correlation, except for latter September and the early part of October, indicates good agreement between our instruments and techniques. As a point of interest, the offset between NBS and GSFC measurements which occurred in September appears to be caused by an unexplained shift in the frequency of the cesium beam oscillator, S-214. The GSFC measurements are based on the "nameplate data" frequency of this oscillator. The rms of the differences between NBS and GSFC monthly means for the months of October 1962 through February 1963 is 7.8 parts

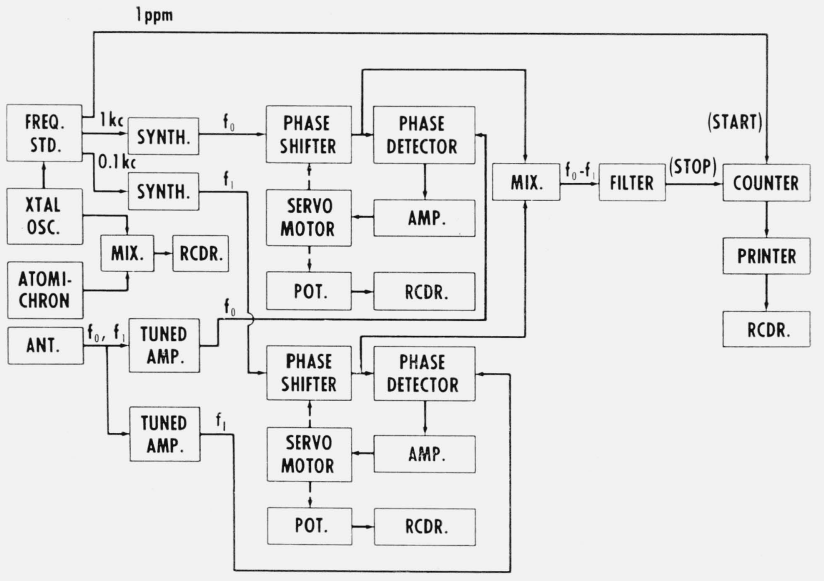

Figure 3. VLF comparison instrumentation.

in $10^{11}$ and the rms deviation from 7.8 is 1.4 parts in $10^{11}$, again demonstrating the high degree of repeatibility of these measurements. Figure 3 is a block diagram of the instrumentation used in the VLF measurements.

One attribute of VLF propagation not yet developed, is its utility for time transmissions. The narrow bandwidth transmitting antennas, necessary to radiate a reasonable percentage of transmitter power, effectively limit pulsed transmissions to rise times in excess of several milliseconds and thus preclude pulse codes from realizing the full utility of VLF propagation stability for timing purposes. An alternative scheme, suggested by A. H. Morgan, of the NBS, is to transmit more than one frequency and utilize their difference frequencies to resolve the inherent cyclic ambiguities in normal VLF transmissions. For the past several months, experimental transmissions of $20.0 \mathrm{kc} / \mathrm{s}$ and $19.9 \mathrm{kc} / \mathrm{s}$ on alternate seconds have been provided by WWVL. Figure 3 shows two separate TRF receivers used to reconstruct the 19.9 and $20.0 \mathrm{kc} / \mathrm{s}$ signals. Although the records of phase shifter position could be used to determine the relative $100 \mathrm{c} / \mathrm{s}$ phase angle, more accuracy is realized by mixing the two phase locked signals and then measuring the phase angle of the resultant $100 \mathrm{c} / \mathrm{s}$ signal. Any drifts or discontinuities in the synthesizers or phase shifters will thus be removed and superior results achieved. If the $19.9 \mathrm{kc} / \mathrm{s}$ transmission were properly phased with respect to the $20.0 \mathrm{kc} / \mathrm{s}$ signal, a particular point, e.g., the positive-going zero crossing, of the $100 \mathrm{c} / \mathrm{s}$ beat note would correspond to the beginning of each second. The present transmissions are not so adjusted and may be used only for system development and testing. Figure 4 is a record of $80 \mathrm{hr}$ of $100 \mathrm{c} / \mathrm{s}$ phase comparison taken at GSFC on July 9, 10, 11, and 12, 1963 . The short term stability is certainly better than $0.1 \mathrm{msec}$; however, the day-to-day stability is only 0.1 to $0.2 \mathrm{msec}$. These drifts could be due to differential propagation delay variations, drifts in the $19.9 \mathrm{kc} / \mathrm{s}$ transmitter synthesizer, just plain noise due to low signal 

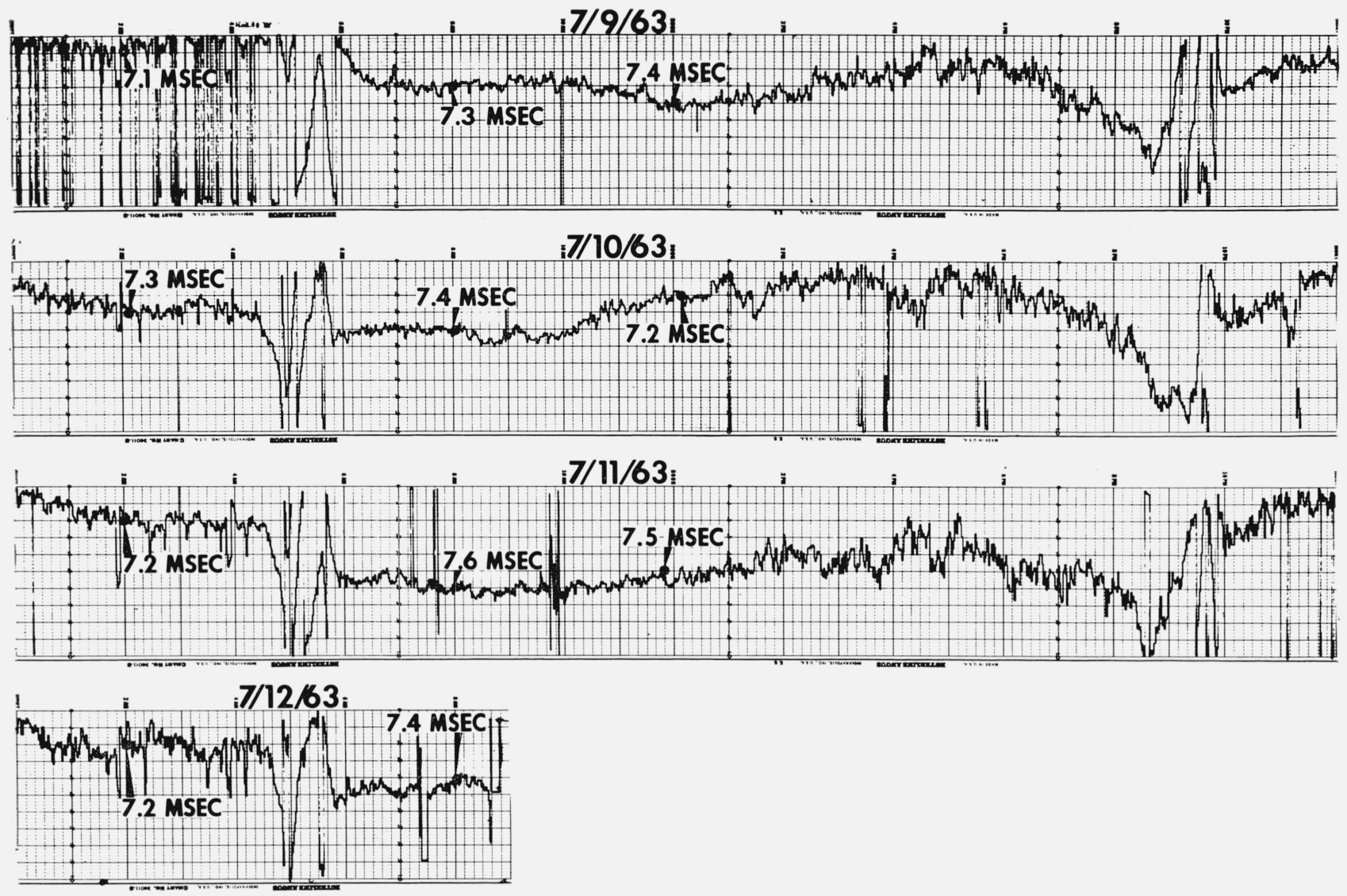

Figure 4. WWVL $100 \mathrm{c} / \mathrm{s}$ phase angle, GSFC July 1963.

power, or drifts in the receiver system. During the same time period, two commercial superheterodyne VLF receivers were used to obtain the $100 \mathrm{c} / \mathrm{s}$ signal by mixing the local oscillator signals: 21.0 and 20.9 $\mathrm{kc} / \mathrm{s}$. Although the results from this arrangement were generally consistent with the GSFC receiver data, certain differences were interesting. The phase noise on the $100 \mathrm{c} / \mathrm{s}$ measurements was larger with the commercial receivers by a factor of from 3 to 5 apparently due to a similar difference in integration times. The commercial receiver system has additional $1 / 2 \mathrm{msec}$ ambiguities on a one-to-one correspondence with cyclic changes of the $19.9 \mathrm{kc} / \mathrm{s}$ or $20.0 \mathrm{kc} / \mathrm{s}$ signal due to the necessity for approximately one-twentieth of an additional cycle of phase change required of the local oscillator in order to make the 19.9 or $20.0 \mathrm{kc} / \mathrm{s}$ signal change by one cycle. These $1 / 2$ msec ambiguities point up the calibration problems of this type of timing system. First, all system filter lags must be known; second, the $10 \mathrm{msec}$ ambiguities of the $100 \mathrm{c} / \mathrm{s}$ signal must be resolved by appropriate transmission coding or using WWV; third, the $50 \mu$ sec ambiguities of the $20 \mathrm{kc} / \mathrm{s}$ signal must be resolved with the $100 \mathrm{c} / \mathrm{s}$ signal. After all these tasks are completed, the propagation time must be known in order to complete the timing of the station. Although this time may be calculated, the accuracy is insufficient; therefore, a portable time standard should be transported from a timed station such as Greenbelt, Md., to the field station (and return) in order to measure the propagation time precisely.

It now appears likely that all Minitrack Stations will be equipped with single VLF receivers by the middle of 1964 . A second receiver will be added the next year in order to provide for simultaneous recordings of two stations, to provide for use of the $20.0 \mathrm{kc} / \mathrm{s}$ and $19.9 \mathrm{kc} / \mathrm{s}$ (or $20.1 \mathrm{kc} / \mathrm{s}$ ) WWVL transmissions, and for backup purposes. Present plans are for the VLF receivers to be used as measurement tools and not as automatic station oscillator correction systems.

Scientists at NASA are looking forward with considerable enthusiasm to working with the much larger $20 \mathrm{kc} / \mathrm{s}$ signal power especially as an aid to separation of the various factors presently causing. unexplained variations in phase angle of the $100 \mathrm{c} / \mathrm{s}$ beat note. We are convinced that VLF time transmission is possible, and the developments in this field now made practical by the new station should be of considerable interest to standards laboratories around the world.

(Paper 68D1-315) 